\title{
Isotropic ion distribution functions triggered by consecutive solar wind bulk velocity jumps: a new equilibrium state
}

\author{
H.-J. Fahr and M. Siewert
}

\begin{abstract}
Argelander Institut für Astronomie der Universität Bonn, Abteilung f. Astrophysik und Extraterrestrische Forschung, Auf dem Huegel 71, 53121 Bonn, Germany

e-mail: msiewert@astro.uni-bonn.de
\end{abstract}

Received 20 August 2010 / Accepted 9 December 2010

\begin{abstract}
Context. Throughout the heliosphere, ion power spectra have been found in observations to exhibit suprathermal tails that follow power laws. Ion power-law spectra, though having a broad range of spectral indices $4.4 \leq \gamma_{v} \leq 6.6$, perhaps favourably seem to have velocity power indices of $\gamma_{v} \simeq(-5)$, a phenomenon that can more or less ubiquitously be found in heliospheric space plasmas. This is probably indicative of an as yet unidentified quasi-equilibrium state of collisionless space plasmas.

Aims. We develop the idea that these forms of ion spectra could be produced by a continuous back- and forth- shuffling of wind convected ions in consecutive jumps from fast to slow, and vice-versa, bulk velocity regimes.

Methods. The appearance of a quasi-equilibrium state due to this shuffling and re-shuffling, as we show, naturally results in ion distribution functions that are superpositions of a series of weighted Maxwellians resulting into power laws beyond a critical velocity border. Spectral intensities thereby anticorrelate with bulk velocities, but power indices depend only slightly on bulk velocity.

Results. The fully developed equilibrium state is shown here, however, to be characterized by a power law with power index $\gamma_{v}=(-3)$, instead of $4.4 \leq \gamma_{v} \leq 6.6$. These latter states, which generally show up in observations, thus may characterize an off-equilibrium state as we discuss in this paper.
\end{abstract}

Key words. shock waves - plasmas - magnetohydrodynamics (MHD) - solar wind

\section{Introduction}

Many observers have obtained comprehensive in-situ spacecraft data of ion distribution functions in near and far interplanetary space, almost consistently confirming that interplanetary ion energy distribution functions in specific energy ranges clearly follow power-law distributions. The relation between differential flux $j(E)$ and distribution function $f(E)$ is given in the form $j(E)=2 E f(E) / m^{2}$, where $E$ and $m$ are the energy and mass of the ion, which makes it clearly evident that a power-law distribution of $f(v) \sim v^{-\kappa_{v}}$ (after applying the relation $E=m v^{2} / 2$ ) is identical to a differential flux function $j(E) \sim E^{-\kappa_{E}} \sim E^{-\left(\kappa_{v} / 2-1\right)}$. An interesting discovery by VOYAGER-1/-2 (Decker et al. 2005, 2008) was that in both the supersonic solar wind regime upstream from the termination shock and the subsonic regime downstream, differential intensities were fairly consistent with an energy power index of $\kappa_{E} \simeq 1.5$ yielding a velocity power index of $\kappa_{v}=5$. Although this index $\kappa_{v}=5$ has often been confirmed for ion distributions in the inner heliosphere (Gloeckler 2003; Fisk \& Gloeckler 2006, 2008; Dayeh et al. 2009a,b), there is still doubt as to whether it can be called an ubiquitously valid power index, since many deviations from that index have been found, especially for ions heavier than protons.

Hill et al. (2009) found for $\mathrm{He}^{+}$ions steeper spectra with $\kappa_{v} \geq 5$. Dayeh et al. (2009b) studied heavy ion distributions (e.g. $\mathrm{C}, \mathrm{O}, \mathrm{Fe}$ ) in interplanetary space at energies between 0.04 and $2.56 \mathrm{MeV} /$ nucleon and found suprathermal tails with spectral indices $\kappa_{E}$ between 1.27 and 2.29 , corresponding to velocity indices of $4.5 \leq \kappa_{v} \leq 6.6$ with a tendency for heavier ion suprathermal tails to be harder than lighter ion tails. This leads to the puzzling observational situation that though suprathermal ion tails often show power laws with $\kappa_{v} \simeq 5$, they also often exhibit significant deviations from that index. Theoreticians searching for explanations of these fairly unclear observational situations thus have to very carefully study conditions under which these ion spectral features have been observed.

Extended tails of ion distribution functions are most often ascribed to the interaction of these ions with different forms of compressive plasma turbulence connected with fluctuations $\delta U$ in the local bulk velocity $U$ of the background wind. This phenomenon was originally described as transit-time damping (see Fisk 1976; Toptygin 1983; Fichtner et al. 1996; le Roux \& Fichtner 1999; Chalov et al. 1997; Chalov 2005).

Fisk \& Gloeckler $(2006,2007,2008)$ attempted to describe the appearance of energetic ion tails with the help of the phasespace transport equation derived by Parker (1965) as a stationary quasi-equilibrium state of a plasma interacting with compressive turbulence in thermally isolated systems. They consider how stochastic fluctuations of the bulk velocity of the background plasma lead to a well-tuned energy diffusion effect composed of adiabatic pumping in the core and spatial diffusion leaving through the tail. However, since in their approaches, the correct choice of system boundary conditions and construction of ensemble averages, as a statistical representation of different realisations of ion distribution functions, is contraversial (see Jokipii \& Lee 2010), we present in this paper an independent, perhaps complementary study of the effect of bulk velocity fluctuations on the shape of ion distribution functions.

To study the phase-space behaviour of ions at their reintegration from one wind frame with velocity $U$ into another frame with $U^{*}$, it is helpful to use some auxiliary physical 
quantities such as phase-space particle invariants. From basic plasma physics, It is well known that in the absence of stochastic processes, such as collisions or wave-particle interactions ions moving along magnetic fields with a non-zero field-magnitude gradient along the field line, behave such that they conserve a dynamic quantity called their magnetic moment $\Gamma=(\mathrm{m} / 2) v_{\perp}^{2} / B$ (see e.g. Treumann \& Baumjohann 1997). Furthermore, we note that this quantity $\Gamma$ also plays the role of an invariant under conditions when ions are co-convected with a plasma bulk containing a frozen-in magnetic field, a typical condition for ions convected by the solar wind.

In the direction of the bulk flow, if the frozen-in magnetic field magnitude decreases, as for the Archimedian spiral field in the inner heliosphere (see Parker 1958; Forsyth et al. 2002), then it can be shown that bulk-convected ions conserve their magnetic moment $\Gamma$ (see Fahr 2007; Fahr \& Siewert 2008; Fahr \& Siewert $2010 \mathrm{~b}$ ). The only restriction hereby is that typical periods $\tau_{B}$ of the field magnitude changes are large compared to the ion gyroperiods $\tau_{\mathrm{g}}$, which guarantees that an adiabatic adaptation to changes in the field magnitude by the gyrating ions can occur.

In addition, the differential motions of ions parallel to frozenin fields, for bulk velocity gradients parallel to the field, exhibit a quantity that can serve as a second particle invariant $\Gamma_{\|}=v_{\|} B / \rho$ (Fahr \& Siewert 2008; Siewert \& Fahr 2008) closely related to the well-known second plasma invariant in the CGL-theory $\Gamma_{\mathrm{CGL}, 2}=P_{\|} B^{2} / \rho^{3}$ (Chew et al. 1956).

These ion invariants cannot only profitably be used to describe the change in the dynamical properties of ions at their passage during the solar wind termination shock (see Fahr \& Siewert 2008, 2009; Fahr \& Siewert 2010b), but are also applicable to any travelling bulk velocity jumps, when transit times $\tau_{\mathrm{t}}=\Delta L / \Delta U$ are much shorter than spatial diffusion times $\tau_{\text {dif }} \simeq 3 L_{U}^{2} / v \Lambda$ (see Toptygin 1983; Chalov et al. 1997). Hereby $\Delta L$ is the extent of the transition region from one to the other bulk velocity, i.e. from $U_{1}$ to $U_{2}, \Delta U=U_{1}-U_{2}$ denotes the bulk velocity jump, $L_{U}$ is the scale of a mono-bulk domain, $v$ is the ion velocity, and $\Lambda$ is the ion mean free path with respect to wave scattering.

At a standing shock an abrupt decrease in the solar wind bulk velocity connected to an increase in the ion densities and frozenin magnetic field magnitudes occurs. The description turns out to be fairly similar for the change in ion distribution functions at travelling bulk velocity jumps wherever they may occur. We now study this context further down in this paper.

\section{Theoretical treatment of stationary shocks}

We begin with the requirements motivated above and quantitatively formulated in Siewert \& Fahr (2008). When applied to two adjacent regions 1 and 2 of different bulk velocities $U_{1}$ and $U_{2}$, one can identify two possibly conserved properties. First, there is the classical electrodynamic invariant, the magnetic moment,

$$
\frac{v_{\perp}^{2}}{B}=\frac{v_{\perp 1}^{2}}{B_{1}}=\frac{v_{\perp 2}^{2}}{B_{2}}=\text { const. }
$$

and second, there is the differential bulk drift

$$
\frac{v_{\|} B}{\rho}=\frac{v_{\| 1} B_{1}}{\rho_{1}}=\frac{v_{\|_{2}} B_{2}}{\rho_{2}}=\text { const. }
$$

These invariants can be considered valid as long as diffusion times are much longer than transit times, i.e. $\tau_{\mathrm{t}} / \tau_{\mathrm{dif}}=$ $v \Lambda \Delta L / 3 L_{U}^{2} \Delta U \ll 1$. Therefore, this condition may become invalid for large ion velocities with $v_{\mathrm{c}}=3 L_{U}^{2} \Delta U / \Lambda \Delta L$, perhaps restricting the validity of the following derivations of this paper to ions with sub-critical velocities $v \leq v_{\mathrm{c}}$. We study this in more detail later in the paper.

\subsection{Individual particle velocites at a stationary shock}

We first study a general standing shock-like MHD feature with a surface normal $\boldsymbol{n}$ pointing antiparallel to the solar wind flow vector $\boldsymbol{U}$, i.e. antiradial, while the wind flows in the radial direction. Introducing the angle $\theta=\angle(\boldsymbol{n}, \boldsymbol{U})$, this corresponds to $\theta=180^{\circ}$. From existing solar wind data taken at varying solar wind bulk velocities, it follows that mass flow continuity is fulfilled essentially in the form of a causally connected one-dimensional flow without sources (see McComas et al. 2000, 2003; Richardson et al. 2003), which may be described by the following relation at larger solar distances

$$
\rho_{1} \boldsymbol{U}_{1}=\rho_{2} \boldsymbol{U}_{2}
$$

i.e. the solar wind behaves as a source-free connection of flows fulfilling mass-flow conservation. Here the parameters $U_{1,2}$ denote fast and slow bulk velocities upstream and downstream of an MHD shock with $\theta=180^{\circ}$, or, more generally, the normal bulk velocity components upstream and downstream in the case of $0^{\circ}<\theta<180^{\circ}$, which is given e.g., at the solar wind termination shock.

At the (anti-)parallel shock, the kinetic invariants may be expressed as a function of the magnetic field pitch angle $\alpha=$ $\angle(\boldsymbol{B}, \boldsymbol{n})=\angle(\boldsymbol{B}, \boldsymbol{U})$. In this case, it follows from the full MHD jump conditions that (see e.g. Gombosi 1998; Erkaev et al. 2000; Diver 2001)

$$
B_{n 1}=B_{n 2}=\text { const. }
$$

and

$$
B_{t 2}=s B_{t 1}
$$

with the compression ratio $s=\rho_{2} / \rho_{1}=U_{1} / U_{2}$. Using these relations, the individual particle velocity components on the upstream and downstream sides may be transformed into each other by

$$
\begin{aligned}
v_{\perp 2}^{2} & =v_{\perp 1}^{2} \frac{B_{2}}{B_{1}} \\
& =v_{\perp 1}^{2} \sqrt{\frac{B_{1}^{2} \cos ^{2} \alpha+s^{2} B_{1}^{2} \sin ^{2} \alpha}{B_{1}^{2} \cos ^{2} \alpha+B_{1}^{2} \sin ^{2} \alpha}} \\
& =v_{\perp 1}^{2} \sqrt{\cos ^{2} \alpha_{1}+s^{2} \sin ^{2} \alpha_{1}}
\end{aligned}
$$

and

$$
\begin{aligned}
v_{\| 2}^{2} & =v_{\| 1}^{2}\left(\frac{B_{1} \rho_{2}}{B_{2} \rho_{1}}\right)^{2} \\
& =v_{\| 1}^{2} \frac{s^{2}}{\cos ^{2} \alpha_{1}+s^{2} \sin ^{2} \alpha_{1}} .
\end{aligned}
$$

To simplify further evaluations, we introduce the abbreviations

$$
A(\alpha)=\sqrt{\cos ^{2} \alpha+s^{2} \sin ^{2} \alpha}
$$

and

$$
B(\alpha)=\frac{s^{2}}{\cos ^{2} \alpha+s^{2} \sin ^{2} \alpha}=\frac{s^{2}}{A^{2}(\alpha)},
$$


i.e. $v_{\perp 2}^{2}=A(\alpha) v_{\perp 1}^{2}$ and $v_{\| 2}^{2}=B(\alpha) v_{\| 1}^{2}$. Combining both results, which results in we find that

$$
\begin{aligned}
v_{2}^{2} & =v_{\perp 2}^{2}+v_{\| 2}^{2} \\
& =v_{\perp 1}^{2} A(\alpha)+v_{\| 1}^{2} B(\alpha) .
\end{aligned}
$$

Introducing the ion pitch angle $\beta=\angle(\boldsymbol{v}, \boldsymbol{n})$, we obtain

$$
\begin{aligned}
v_{\perp 2}^{2} & =v_{2}^{2} \sin ^{2} \beta_{2}=v_{1}^{2} \sin ^{2} \beta_{1} A\left(\alpha_{1}\right), \\
v_{\| 2}^{2} & =v_{2}^{2} \cos ^{2} \beta_{2}=v_{1}^{2} \cos ^{2} \beta_{1} B\left(\alpha_{1}\right),
\end{aligned}
$$

and

$$
v_{2}^{2}=v_{1}^{2}\left[\sin ^{2} \beta_{1} A(\alpha)+\cos ^{2} \beta_{1} B(\alpha)\right],
$$

which allows us to derive the new dynamically associated downstream ion pitch-angle $\beta_{2}$ as a function of upstream parameters

$$
\operatorname{ctg}^{2} \beta_{2}=\operatorname{ctg}^{2} \beta_{1} \cdot \frac{B\left(\alpha_{1}\right)}{A\left(\alpha_{1}\right)}=\operatorname{ctg}^{2} \beta_{1} \cdot \frac{s^{2}}{A^{3}\left(\alpha_{1}\right)} .
$$

Another simplification that we use in the following sections is

$$
v_{2}^{2}=C v_{1}^{2},
$$

i.e.

$$
C=C_{\text {aniso }}=\sin ^{2} \beta_{1} A(\alpha)+\cos ^{2} \beta_{1} B(\alpha) .
$$

\subsection{The distribution function at standing shocks}

Using these results for individual particles, we derive the downstream distribution function. Using Eqs. (11) and (12) and also Liouville's equation (i.e. continuity of the differential phase space flux at the jump), the connection between the distribution functions upstream and downstream can be derived to be

$$
2 \pi U_{2} f_{2}^{<} \mathrm{d} v_{2} v_{2}^{2} \sin \beta_{2} \mathrm{~d} \beta_{2}=2 \pi U_{1} f_{1} \mathrm{~d} v_{1} v_{1}^{2} \sin \beta_{1} \mathrm{~d} \beta_{1},
$$

where $f_{2}^{<}=f_{2}^{<}\left(v_{2}, \beta_{2}\right)$ is the initial downstream ion distribution function, before pitch-angle isotropisation starts to dominate the system. This intermediate distribution function is in most cases unstable with respect to plasma instabilities and will trigger wave generation (Fahr \& Siewert 2007, 2008; Siewert \& Fahr 2008; Fahr \& Siewert 2010b). From Eqs. (13) and (17), it then follows that $f_{2}^{<}\left(v_{2}, \beta_{2}\right)$ may be expressed by

$$
f_{2}^{<}\left(v_{2}, \beta_{2}\right)=f_{1}\left(\boldsymbol{v}_{1}\right) \frac{U_{1}}{U_{2}} \frac{v_{1}^{2}}{v_{2}^{2}} \frac{\mathrm{d} v_{1}}{\mathrm{~d} v_{2}} \frac{\sin \beta_{1} \mathrm{~d} \beta_{1}}{\sin \beta_{2} \mathrm{~d} \beta_{2}} .
$$

From Eq. (11), it follows that

$$
\frac{\sin \beta_{1}}{\sin \beta_{2}}=v_{2} / v_{1} \frac{1}{\sqrt{A(\alpha)}},
$$

while differentiating Eq. (14) results in

$$
\operatorname{ctg} \beta_{2}=\operatorname{ctg} \beta_{1} \sqrt{B(\alpha) / A(\alpha)},
$$

or

$$
\frac{\mathrm{d}}{\mathrm{d} \beta_{2}} \operatorname{ctg} \beta_{2}=\frac{\mathrm{d}}{\mathrm{d} \beta_{1}} \operatorname{ctg} \beta_{1} \frac{\mathrm{d} \beta_{1}}{\mathrm{~d} \beta_{2}} \sqrt{B(\alpha) / A(\alpha)},
$$

$$
\begin{aligned}
\frac{\sin \beta_{1} \mathrm{~d} \beta_{1}}{\sin \beta_{2} \mathrm{~d} \beta_{2}} & =\frac{\sin ^{3} \beta_{1}}{\sin ^{3} \beta_{2}} \sqrt{\frac{A(\alpha)}{B(\alpha)}} \\
& =\frac{v_{2}^{3}}{v_{1}^{3}} \frac{1}{A^{3 / 2}(\alpha)} \sqrt{\frac{A(\alpha)}{B(\alpha)}} \\
& =\frac{v_{2}^{3}}{v_{1}^{3}} \frac{1}{A(\alpha)} \sqrt{\frac{1}{B(\alpha)}} .
\end{aligned}
$$

Therefore, Eq. (18) transforms into

$$
f_{2}^{<}\left(v_{2}, \beta_{2}\right)=\frac{1}{A\left(\alpha_{1}\right) \sqrt{B\left(\alpha_{1}\right.}} f_{1}\left(\boldsymbol{v}_{1}\right) \frac{U_{1}}{U_{2}} \frac{v_{2}}{v_{1}} \frac{\mathrm{d} v_{1}}{\mathrm{~d} v_{2}} .
$$

Finally, using Eqs. (5) and (13) we find that

$$
\begin{aligned}
f_{2}^{<}\left(v_{2}, \beta_{2}\right) & =f_{1}\left(v_{1}\left(v_{2}, \beta_{2}\right), \beta_{1}\left(v_{2}, \beta_{2}\right)\right) \frac{s}{A\left(\alpha_{1}\right) \sqrt{B\left(\alpha_{1}\right)}} \\
& =f_{1}\left(v_{1}\left(v_{2}, \beta_{2}\right), \beta_{1}\left(v_{2}, \beta_{2}\right)\right),
\end{aligned}
$$

where we have used Eqs. (8) and (9), from which it follows that $A \sqrt{B}=s$. In other words, only the phase space variables and not the distribution function itself are modified for the shock, which agrees with earlier results by Siewert \& Fahr (2008).

\subsection{Isotropic distributions}

An even simpler form of this expression may be derived by assuming that $f_{2}^{<}$quickly returns to exhibiting pitch-angle isotropy because of effective pitch-angle scattering processes on both sides of the jump. Using these additional assumptions, the velocity transformation (Eq. (18)) takes the form

$$
\begin{aligned}
v_{2}^{2} & =v_{1}^{2} \frac{1}{2} \int_{0}^{\pi} \sin \beta \mathrm{d} \beta\left[\sin ^{2} \beta A\left(\alpha_{1}\right)+\cos ^{2} \beta B\left(\alpha_{1}\right)\right] \\
& =v_{1}^{2}\left[\frac{2}{3} A\left(\alpha_{1}\right)+\frac{1}{3} B\left(\alpha_{1}\right)\right] .
\end{aligned}
$$

Introducing, again, a factor of C (analoguous to Eq. (16)), we obtain

$$
C_{\text {iso }}(\alpha)=\frac{2}{3} A(\alpha)+\frac{1}{3} B(\alpha) .
$$

This result then allows us to write down a generalised formulation of the Liouville equation (similar to Eq. (17), but for isotropic distributions)

$$
4 \pi U_{2} f_{2}\left(v_{2}\right) v_{2}^{2} \mathrm{~d} v_{2}=4 \pi U_{1} f_{1}\left(v_{1}\right) v_{1}^{2} \mathrm{~d} v_{1} .
$$

From this equation, it easily follows that

$$
f_{2}\left(v_{2}\right)=f_{1}\left(v_{1}\right) \frac{U_{1}}{U_{2}} \frac{\mathrm{d} v_{1}}{\mathrm{~d} v_{2}} \frac{v_{1}^{2}}{v_{2}^{2}}
$$

and further that

$$
f_{2}\left(v_{2}\right)=f_{1}\left(\frac{v_{2}}{\sqrt{C_{\text {iso }}\left(\alpha_{1}\right)}}\right) \frac{s}{C_{\text {iso }}^{3 / 2}} .
$$




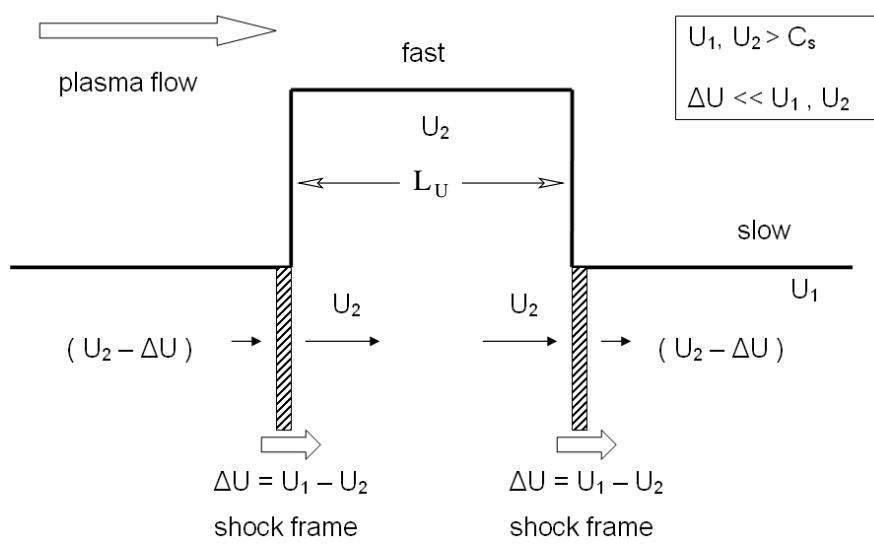

Fig. 1. The general form of a travelling shock induced by a nonlinear electrostatic double-soliton structure.

\section{Theoretical treatment of travelling shocks}

\subsection{A toy model for consecutive travelling bulk velocity jumps}

We now attempt to apply the result derived above to the fairly equivalent situation of travelling shocks, i.e. travelling structures of bulk velocity jumps in the solar wind (e.g. see observations presented by Richardson et al. 2008).

We assume a jump contour (sketched in Fig. 1) with an upstream bulk velocity $U_{1}$ and a downstream velocity $U_{2}$, which in contrast to classical standing shock waves, are both assumed to be supersonic, i.e., $U_{1}, U_{2} \gg c_{\mathrm{s}}$, and that $\Delta U=U_{1}-U_{2} \ll$ $c_{\mathrm{s}} \ll U_{1}, U_{2}$ is the velocity at which the structure moves outwards with the solar wind. In this configuration, we now have travelling shock frames with a propagating static jump structure, i.e., frames moving with a velocity $\Delta U=U_{1}-U_{2}$, where at synchronously moving boundaries two opposite types of jumps by $\Delta U= \pm U$ can occur.

These bulk velocity jumps may physically be envisioned to be caused by a nonlinear electrostatic soliton composed of a superposition of electrostatic waves for which $\boldsymbol{k}\|\boldsymbol{E}\| \boldsymbol{U}$ with dispersion and dissipation (see Spatschek 1990; Infeld \& Rowlands 1990) that propagates with a velocity $\Delta U$ through a monokinetic plasma bulk flow. Wherever this soliton arrives, it triggers a bulk velocity jump according to

$$
\Delta U^{2}=\frac{2 e}{m} \int_{r_{1}}^{r_{2}} E(r) \mathrm{d} r,
$$

over a distance increment of $\Delta r=r_{2}-r_{1}$. The opposite soliton with $\boldsymbol{E}^{*}\left(r-\mu_{\mathrm{S}} t\right)=-\boldsymbol{E}\left(r-\mu_{\mathrm{S}} t\right)$ and an identical wave profile propagates with the same group velocity $\mu_{\mathrm{S}}=\Delta U$, but wherever it arrives, it decelerates the plasma flow by $\Delta U^{*}=-\Delta U$ switching the bulk velocity back to its earlier value.

For demonstration purposes, we adopt jump conditions which we use in the following sections of this paper given by

$$
U_{1}=450 \mathrm{~km} \mathrm{~s}^{-1}, \quad U_{2}=400 \mathrm{~km} \mathrm{~s}^{-1}, \quad \Delta U=50 \mathrm{~km} \mathrm{~s}^{-1} .
$$

\subsection{Evolution of distribution functions at repeated travelling shock passings}

Using our earlier results for standing shock waves, it is trivial to generalise Eq. (24) to moving shock waves. Repeating our earlier results, the intermediate (pre-isotropisation) downstream distribution function is given by

$$
f_{\mathrm{s}}^{1,<}\left(v_{\mathrm{s}}, \beta_{\mathrm{s}}\right)=f_{\mathrm{f}}\left(v_{\mathrm{f}}\left(v_{\mathrm{s}}, \beta_{\mathrm{s}}\right), \beta_{\mathrm{f}}\left(v_{\mathrm{s}}, \beta_{\mathrm{s}}\right)\right),
$$

where $s_{\mathrm{fs}}$ is the fast-to-slow compression ratio defined by

$$
s_{\mathrm{fs}}=U_{\mathrm{f}} / U_{\mathrm{s}}=\frac{(U+\Delta U)}{(U-\Delta U)} .
$$

After some time $\tau=L / \Delta U$, this distribution function will be re-integrated (or back-shuffled) into the fast bulk motion $U_{\mathrm{f}}$ at the position of the reverse travelling shock (i.e. induced by the reverse electrostatic soliton). Requesting again the conservation of the two kinetic invariants, we may apply our formalism again and now obtain

$$
f_{\mathrm{f}}^{1,<}\left(v_{\mathrm{f}}, \beta_{\mathrm{f}}\right)=\cdot f_{\mathrm{s}}^{1,<}\left(v_{\mathrm{s}}, \beta_{\mathrm{s}}\right),
$$

where we have introduced to convert $\boldsymbol{v}_{\mathrm{s}}$ again into $\boldsymbol{v}_{\mathrm{f}}$ the slowto-fast compression ratio (see also Sect. 4)

$$
s_{\mathrm{sf}}=s_{\mathrm{fs}}^{-1} \text {. }
$$

As we demonstrate in Appendix A, the velocity components after crossing both shock structures remain identical, i.e. particles return to their initial phase space position after crossing the double structure, if the dynamical invariants given by Eqs. (1) and (2) are conserved at both passages and in-between. Therefore, no significant modification of the initial distribution function is expected after $n$ passings if the invariants are constantly conserved (but see Sect. 4.4 for the description of a mechanism to change this).

A different result is obtained for the isotropic, pitch-angle scattering-processed distribution function, (i.e. jump passage and isotropisation). Using Eq. (29), we obtain

$$
\begin{aligned}
f_{f, 1}\left(v_{\mathrm{f}}\right) & =f_{\mathrm{s}}^{1}\left(\frac{v_{\mathrm{s}}}{\sqrt{C_{\mathrm{iso}}\left(\alpha_{\mathrm{s}}\right)}}\right) \frac{s_{\mathrm{sf}}}{C_{\mathrm{iso}}\left(\alpha_{\mathrm{s}}\right)} \\
& =f_{f, 0}\left(\frac{v_{\mathrm{f}}}{\sqrt{C_{\mathrm{iso}}\left(\alpha_{\mathrm{s}}\right) C_{\mathrm{iso}}\left(\alpha_{\mathrm{f}}\right)}}\right) \frac{1}{C_{\text {iso }}\left(\alpha_{\mathrm{s}}\right) C_{\text {iso }}\left(\alpha_{\mathrm{f}}\right)} .
\end{aligned}
$$

This result is different from the anisotropic case and results in an observable modification of the reconverted ion distribution function with respect to the initial one. This result may be generalised to an arbitrary number of repeating soliton-antisoliton crossings by noting that the results for one twin-structure crossing are essentially identical to those for the $i$-th crossing in a long chain of soliton-antisoliton passages, i.e.

$$
f_{\mathrm{f}, i}\left(v_{\mathrm{f}}\right)=f_{\mathrm{f}, i-1}\left(\frac{v_{\mathrm{f}}^{i}}{C_{\text {iso }}\left(\alpha_{\mathrm{f}}\right) C_{\text {iso }}\left(\alpha_{\mathrm{s}}\right)}\right) \frac{1}{C_{\text {iso }}\left(\alpha_{\mathrm{f}}\right) C_{\text {iso }}\left(\alpha_{\mathrm{s}}\right)} .
$$

This equation can easily be transformed into a non-iterative form for $i$ crossings, resulting in

$$
\begin{aligned}
f_{\mathrm{f}, i}\left(v_{\mathrm{f}}\right)= & f_{\mathrm{f}, i}\left(\frac{v_{\mathrm{f}}}{\left(C_{\text {iso }}\left(\alpha_{\mathrm{f}}\right) C_{\text {iso }}\left(\alpha_{\mathrm{s}}\right)\right)^{i / 2}}\right) \\
& \times \frac{1}{\left(C_{\text {iso }}\left(\alpha_{\mathrm{f}}\right) C_{\text {iso }}\left(\alpha_{\mathrm{s}}\right)\right)^{3 i / 2}} .
\end{aligned}
$$

Equation (37) illustrates an interesting property. The isotropic shock introduces an additional normalising factor, $\left(C\left(\alpha_{\mathrm{f}}\right) C\left(\alpha_{\mathrm{s}}\right)\right)^{-3 i / 2}$, which is always positive and always either larger or smaller than 1 for all $i$. This means that the behaviour of the distribution function (i.e. its narrowing or broadening) can be derived by studying the behaviour of one single iteration.

For completeness, we also derive an equivalent formulation for the slow plasma flow, i.e. the distribution function emerging after $i$ crossings of a double fast-to-slow-to-fast structure, 
followed by one final fast-to-slow crossings. From Eqs. (31) and (37), it then follows that

$$
\begin{aligned}
f_{\mathrm{s}}^{i}\left(v_{\mathrm{f}}\right)= & f_{\mathrm{f}}^{0}\left(\frac{v_{\mathrm{f}}}{C_{\mathrm{iso}}^{(i+1) / 2}\left(\alpha_{\mathrm{f}}\right) C_{\mathrm{iso}}^{i / 2}\left(\alpha_{\mathrm{s}}\right)}\right) \\
& \times \frac{1}{C_{\text {iso }}^{3(i+1) / 2}\left(\alpha_{\mathrm{f}}\right) C_{\mathrm{iso}}^{3 i / 2}\left(\alpha_{\mathrm{s}}\right)} .
\end{aligned}
$$

\section{Study of typical results}

We now study the behaviour of these equations when applying them to typical initial distribution functions. Using as standard values, the velocity fluctuations adopted in Sect. 3.1, we obtain

$$
s_{\mathrm{fs}}=U_{\mathrm{f}} / U_{\mathrm{s}}=\frac{\left(U_{\mathrm{s}}\right)}{\left(U_{\mathrm{s}}-\Delta U\right)}=\frac{400}{400-50}=\frac{8}{7}=1.14
$$

and

$$
s_{\mathrm{sf}}=U_{\mathrm{s}} / U_{\mathrm{f}}=\frac{\left(U_{\mathrm{s}}-\Delta U\right)}{U_{\mathrm{s}}}=\frac{350}{400}=\frac{7}{8}=0.87 .
$$

\subsection{The development of an initial Maxwell-Boltzmann distribution}

We start from an isotropic, upstream Maxwellian in the reference frame of the fast bulk flow, i.e.

$$
f_{\mathrm{f}, 0}=\Phi_{0} \exp \left[-\frac{m v_{\mathrm{f}}^{2}}{2 k T_{\mathrm{f}, 0}}\right]=\Phi_{0} \exp \left[-\Gamma_{\mathrm{f}, 0} v_{\mathrm{f}}^{2}\right],
$$

where the subscript 0 denotes the initial upstream temperature parameter value of the initial ion population, before the first crossing of a jump structure, and $\Phi_{0}$ is the initial normalisation of the Maxwellian. This initial ion population can be represented by pick-up ions implanted into the bulk flow by freshly ionized H-atoms, implying that $T_{\mathrm{f}, 0}$ is given by $\frac{3}{2} k T_{\mathrm{f}, 0}=\frac{1}{2} m U_{\mathrm{f}}^{2}$. (This model results in a configuration where a new initial, "pristine" ion population is generated continuously, and in addition to any non-initial population that has already crossed multiple jump structures.) Equation (37) then leads to the isotropic downstream ion distribution function, after crossing $i$ soliton structures given by

$$
\begin{aligned}
f_{\mathrm{f}, \mathrm{i}}\left(v_{\mathrm{s}}, \beta_{\mathrm{s}}\right)= & \Phi_{0} \exp \left[-\Gamma_{\mathrm{f}} \frac{v_{\mathrm{s}}^{2}}{\left(C_{\mathrm{iso}}\left(\alpha_{\mathrm{f}}\right) C_{\mathrm{iso}}\left(\alpha_{\mathrm{s}}\right)\right)^{i}}\right] \\
& \times \frac{1}{\left(C_{\mathrm{iso}}\left(\alpha_{\mathrm{f}}\right) C_{\mathrm{iso}}\left(\alpha_{\mathrm{s}}\right)\right)^{3 i / 2}} \\
= & \Phi_{\mathrm{i}} \exp \left[-\Gamma_{\mathrm{f}} \frac{v_{\mathrm{s}}^{2}}{\left(C_{\text {iso }}\left(\alpha_{\mathrm{f}}\right) C_{\text {iso }}\left(\alpha_{\mathrm{s}}\right)\right)^{i}}\right],
\end{aligned}
$$

where we have introduced the abbreviations

$$
\Phi_{\mathrm{i}}=\frac{\Phi_{0}}{\left(C_{\text {iso }}\left(\alpha_{\mathrm{f}}\right) C_{\text {iso }}\left(\alpha_{\mathrm{s}}\right)\right)^{3 i / 2}}
$$

and

$$
\Gamma_{\mathrm{f}, \mathrm{i}}=\frac{\Gamma_{\mathrm{f}, 0}}{\left(C_{\mathrm{iso}}\left(\alpha_{\mathrm{f}}\right) C_{\mathrm{iso}}\left(\alpha_{\mathrm{s}}\right)\right)^{i / 2}} .
$$

In other words, since $T \propto \Gamma^{-1}$, we can see that with an increasing normalisation factor, the temperature decreases, i.e. a narrowing distribution function correlates with an increasing maximum, and vice versa.

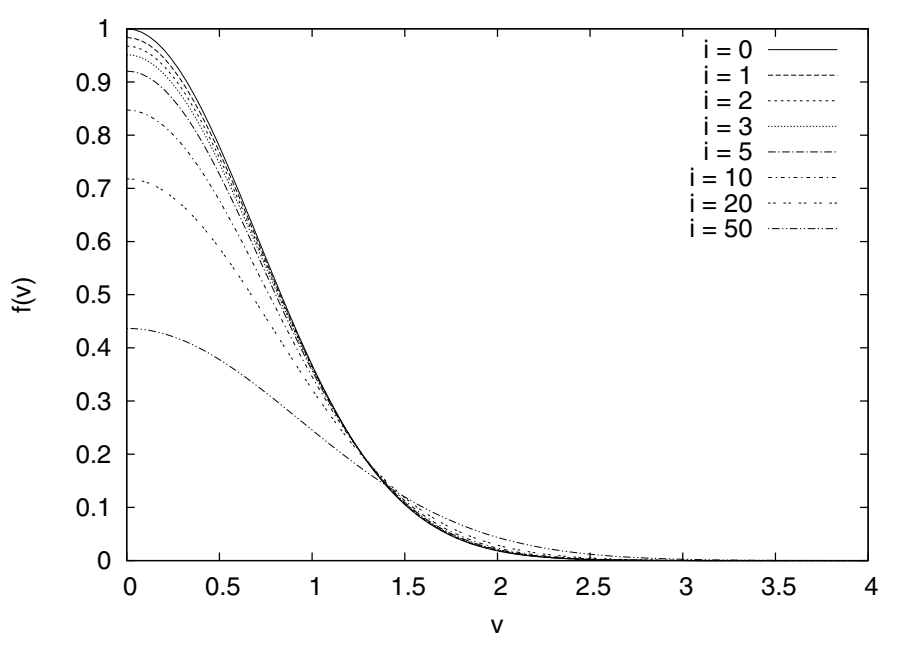

Fig. 2. Distribution functions after $i$ individual pumping processes according to Eq. (37) for a perpendicular magnetic field.

For the standard values adopted in our studies and a perpendicular shock (i.e. $\alpha_{\mathrm{f}}=\alpha_{\mathrm{s}}=90^{\circ}$ ), we find that $C_{\text {iso }}\left(90^{\circ}\right)=$ $\frac{2}{3} s+\frac{1}{3}$, and hence,

$$
\begin{aligned}
\left(C_{\text {iso }}\left(\alpha_{\mathrm{f}}\right) C_{\text {iso }}\left(\alpha_{\mathrm{s}}\right)\right)^{i / 2} & =\left(\frac{5}{9}+\frac{2}{9}\left(s_{\mathrm{fs}}+s_{\mathrm{sf}}\right)\right)^{i / 2} \\
& =\frac{\sqrt{\left(5+2\left(s_{\mathrm{fs}}+s_{\mathrm{sf}}\right)\right)^{i}}}{3^{i}} .
\end{aligned}
$$

Adopting $s_{\mathrm{sf}}=s_{\mathrm{sf}}^{-1}=1$ as a lower limit for $s_{\mathrm{fs}}$, we see that the expression in Eq. (45) is always larger than 1, i.e. the maximum (which coincides with the norm) of the distribution function shrinks as the temperature of the new population grows. Physically, this corresponds to an entropy increase, which demonstrates that the underlying mechanism provides a valid heating process, which might be dubbed "pumped heating".

Typical results for an initial Maxwellian with $\Gamma_{0}=1, \alpha_{\mathrm{f}}=$ $\alpha_{\mathrm{s}}=90^{\circ}$, and $s_{\mathrm{fs}}=1.14$ are presented in Fig. 2, where one can easily observe the temperature increase as the broadening of the distribution function. The evolution of the temperature as a function of the counting index $i$ is given in Fig. 3, while Fig. 4 demonstrates the difference between the initial, slow, and fast distribution functions at one individual double-jump structure.

\subsection{Distribution functions in the presence of continuous reinjection of the initial distribution}

In Sect. 4.1, we introduced charge exchange processes (i.e. pickup ion injection) as a possible source of the initial distribution function. In this configuration, the initial distribution function will always be reinjected, and the final distribution will thus correspond to a sum over Maxwellians of order $i$ that have been processed over a varying amount of time

$$
f_{\mathrm{f}, \text { final }}(v)=\sum_{i} \Phi_{\mathrm{f}, i} \cdot \exp \left[-v^{2} \Gamma_{\mathrm{f}, i}\right] .
$$

If we assume that there is a significant distance between the fastto-slow and the slow-to-fast transitions, then the slow distribution functions also need to be taken into account, resulting in the additional contribution

$$
f_{\mathrm{s}}(v)=s_{\mathrm{fs}} \sum_{i} \frac{\Phi_{\mathrm{s}, i}}{C_{\mathrm{iso}}\left(\alpha_{\mathrm{f}}\right)} \exp \left[-v^{2} \frac{\Gamma_{\mathrm{f}, i}}{\sqrt{C_{\mathrm{iso}}\left(\alpha_{\mathrm{f}}\right)}}\right] .
$$




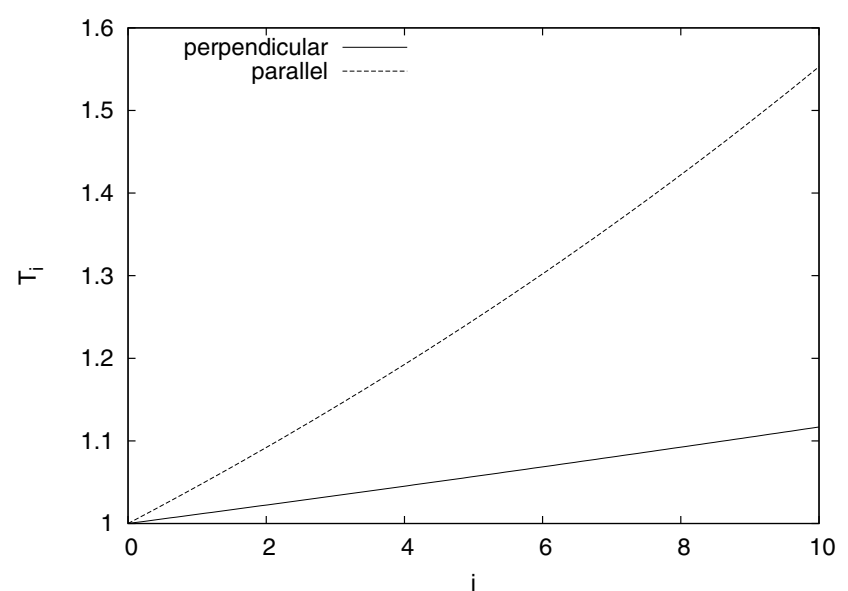

Fig. 3. Temperature parameter $1 . / \Gamma$ as a function of the number of pumping processes for perpendicular and parallel magnetic fields.

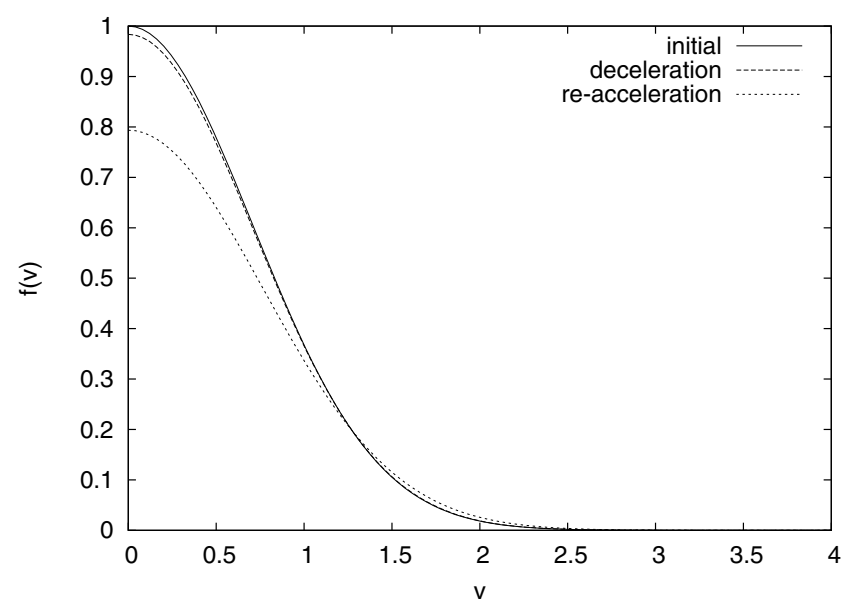

Fig. 4. Distribution functions at a double jump-structure. Depicted are the initial (pristine) function as well as the processed function after crossing the first half (deceleration) and both parts of a double-jump structurere-acceleration).

This interestingly enough reflects the well-established finding in observational data that the spectral intensities of ion distributions are inversely proportional to the bulk velocity of the solar wind (Hill et al. 2009; Dayeh et al. 2009b; Simunac et al. 2009).

In addition, we recall from Schwadron et al. (2010) that a suitable superposition of Maxwellians with an entropymaximising weight function $F(\lambda)=\exp \left(-\lambda / \lambda_{0}\right)$ leads to Kappa functions, and in the limit of $v^{2} \gg \lambda_{0}$ to power laws according to

$$
\begin{aligned}
f\left(v, \lambda_{0}\right) & =n_{0} \int \mathrm{d} \lambda F(\lambda)\left(\frac{\lambda_{0}}{\lambda}\right)^{\nu-s}\left(\frac{\lambda}{\pi}\right)^{3 / 2} \exp \left(-\lambda v^{2}\right) \\
& =n_{0}\left(\frac{\lambda_{0}}{\pi}\right)^{3 / 2} \frac{\Gamma(\kappa+1)}{\left(1+\lambda_{0} v^{2}\right)^{\kappa+1}} .
\end{aligned}
$$

Since $\lambda=\Gamma_{\mathrm{f}, i}$, this requirement for $F(\lambda)$ implies that,

$$
F(\Gamma)=\exp \left(-\frac{\Gamma}{\lambda_{0}}\right) \stackrel{!}{\propto} \Phi(\Gamma)
$$

where we have skipped the subscript $i$ for the conversion between a discrete sum and a continuous integral. Using Eqs. (43) and (44), we find that

$$
\Phi(\Gamma)=\Phi_{0}\left(\frac{\Gamma}{\Gamma_{0}}\right)^{3}
$$

This result differs significantly from the exponential requirement, proving that the mechanism derived here, while physically highly interesting, is unable to provide the configuration required by Schwadron et al. (2010).

\subsection{The development of an initial power-law distribution}

Even though our proposed mechanism does not automatically result in the emergence of power-law distribution functions, it is nevertheless of high interest to study how existing power-law distribution functions are modified by the bulk velocity fluctuations that have been studied before. Starting with an initially isotropic distribution function of the form

$$
f_{0}(v)=\phi_{0} v^{-\kappa}
$$

it follows from Eq. (37) that

$$
\begin{aligned}
f_{\mathrm{f}, i}\left(v_{\mathrm{f}}\right) & =\frac{\phi_{0} v^{-\kappa}}{\left(C_{\text {iso }}\left(\alpha_{\mathrm{f}}\right) C_{\text {iso }}\left(\alpha_{\mathrm{s}}\right)\right)^{-\kappa i / 2}} \frac{1}{\left(C_{\text {iso }}\left(\alpha_{\mathrm{f}}\right) C_{\text {iso }}\left(\alpha_{\mathrm{s}}\right)\right)^{3 i / 2}} \\
& =\frac{\phi_{0}}{\left(C_{\text {iso }}\left(\alpha_{\mathrm{f}}\right) C_{\text {iso }}\left(\alpha_{\mathrm{s}}\right)\right)^{(3-\kappa) i / 2}} v^{-\kappa} .
\end{aligned}
$$

This result proves two different points. First, the powerlaw index will always be conserved, supporting earlier results by Siewert \& Fahr (2008), who proved that power-law indices should be conserved because of the results obtained at the termination shock. The second important consideration is the behaviour of the normalising factor, which is either growing (for $\kappa>3$ ) or shrinking (for $\kappa<3$ ) according to the numerical value of $\kappa$.

\subsection{A new equilibrum state}

We assume that a permanent back- and forth-shuffling of ions from fast-velocity to slow-velocity bulk regimes and back takes place, where the size of the fluctuation $\Delta U$ and the resulting compression ratios $s_{\mathrm{fs}}=s_{\mathrm{sf}}^{-1}$ do not vary signifcantly, which means that a new equilibrium state can be expected to arise from this process. This equilibrium would be reached if the distribution function of the ions is no longer modifiedwhile a crossing of ions from the upstream to downstream and back to the upstream side occurs, i.e. when $f_{i+1}(v)=f_{i}(v)$ is valid, or

$$
f_{i+1}(v)=f^{i}(v)=f^{i}\left(\frac{v}{\sqrt{C\left(\alpha_{\mathrm{f}}\right) C\left(\alpha_{\mathrm{s}}\right)}}\right)\left(C\left(\alpha_{\mathrm{f}}\right) C\left(\alpha_{\mathrm{s}}\right)\right)^{-3 / 2} \text {. }
$$

The requested special and appropriate equilibrum distribution function $f(v)$ is given in implicit form by this above requirement. As suggested by the previous study of power laws, one solution of this equation may be obtained by a power law approach, i.e. $f(v)=n v^{-\kappa}$, which according to Eq. (53) results in the requirement

$$
1=\left(\frac{1}{\sqrt{C_{\text {iso }}\left(\alpha_{\mathrm{f}}\right) C_{\text {iso }}\left(\alpha_{\mathrm{s}}\right)}}\right)^{-\kappa}\left(C_{\text {iso }}\left(\alpha_{\mathrm{f}}\right) C_{\text {iso }}\left(\alpha_{\mathrm{s}}\right)\right)^{-3 / 2}
$$

leading to

$$
1=\left(C_{\text {iso }}\left(\alpha_{\mathrm{f}}\right) C_{\text {iso }}\left(\alpha_{\mathrm{s}}\right)\right)^{-3 / 2+\kappa / 2},
$$

which requires that $\kappa=3$. This result proves that any astrophysical plasma flow encountering multiple shock-like fluctuations will ultimately take the form of a power-law distribution with a power index of $-\kappa=-3$, which, interestingly, neither depends on the fluctuation amplitude $\delta U$ nor the magnetic tilt angles $\alpha$, 
obviously meaning that this equilibrium state is widely insensitive to the specific plasma conditions.

On the other hand, it must clearly be stated that this power index of $-\kappa=-3$ is different from the one most often found in space plasmas (see Gloeckler 2003; Fisk \& Gloeckler 2006, 2007, 2008; Hill et al. 2009; Popecki et al. 2010; Dayeh et al. 2009a,b), namely $-4.4 \geq-\kappa \geq-6.6$. This may perhaps be explained by varying our model assumptions, namely the assumption of rapid isotropisation. This interesting bridge between observations and the above formulated theoretical requirements may be to assume that the ions swept into the new bulk regime do not have enough time to isotropize completely, before they are re-incorporated into the consecutive other bulk, induced by a reverse jump. In this case, we are faced with a distribution function composed of an isotropic part and an anisotropic one. As we demonstrate in Appendix A of this paper, the ions that do not undergoing any pitch-angle isotropisation before being reincorporated into the earlier bulk regime are re-integrated precisely with their original velocity components, i.e. no entropy is generated by this component of the system. Only the ions that have been isotropized before re-integration change the distribution function in the other bulk regime upon re-integration according to the relations developed in the paragraphs above.

We now ask in which sense this envisioned off-equilibrium state may change our earlier results. First, we introduce the isotropisation ratio $\epsilon=n_{\text {iso }} / n_{\text {tot }}$, which defines how many ions successfully undergo the isotropisation process before they encounter the next bulk velocity jump structure. To generate an observable power law, the convergence of the system towards the equilibrum state must be sufficiently fast, i.e. after a finite number of shock transitions. Now, accepting these assumptions, our modified implicit equation is given by

$$
\begin{aligned}
f_{i+1}(v)= & \epsilon f_{i}\left(\frac{v}{\left(C_{\text {iso }}\left(\alpha_{\mathrm{s}}\right) C_{\text {iso }}\left(\alpha_{\mathrm{f}}\right)\right)^{1 / 2}}\right) \\
& \times\left(C_{\text {iso }}\left(\alpha_{\mathrm{f}}\right) C_{\text {iso }}\left(\alpha_{\mathrm{s}}\right)\right)^{-3 / 2}
\end{aligned}
$$

which for a power law with spectral index $\tilde{\kappa}$ leads to the requirement

$$
\frac{1}{\epsilon}=\left(C_{\text {iso }}\left(\alpha_{\mathrm{f}}\right) C_{\text {iso }}\left(\alpha_{\mathrm{s}}\right)\right)^{\frac{\tilde{\kappa}}{2}-\frac{3}{2}}
$$

resulting in the solution

$$
\tilde{\kappa}=3-2 \frac{\lg \epsilon_{i}}{\lg C_{\text {iso }}\left(\alpha_{\mathrm{f}}\right) C_{\text {iso }}\left(\alpha_{\mathrm{s}}\right)} .
$$

This power-law index $\tilde{\kappa}$ is mildly (logarithmically) dependent on the conditions of the bulk jump. Assuming, as an example, a perpendicular jump, i.e. a jump with $\alpha=\pi / 2$ and the standard values given in Sect. 4 (i.e. $s=1.17$ ), it follows from Eq. (45) that $C\left(90^{\circ}\right) C\left(90^{\circ}\right) \simeq 1.0055$. Thus, one finds that

$$
\tilde{\kappa}=3-2 \frac{\ln \epsilon}{\ln (1.0055)} \text {. }
$$

Plotting this equation as a function of $\epsilon$, Fig. 5 then allows us to estimate the conditions under which for residual pitch-angle anisotropies an average value of $\tilde{\kappa} \simeq 5$ is reached. From this figure, it follows that the stable power-law index $\kappa$ strongly on the plasma jump parameters, although in general, only small anisotropy values $0.95 \leq \epsilon \leq 1$ result in power-law indices in the regime of commonly observed values, i.e. $-4.4 \gtrsim-\kappa \gtrsim-6.6$. This result suggests that realistic power-law indices observed in astrophysical systems may be stabilised by local bulk velocity

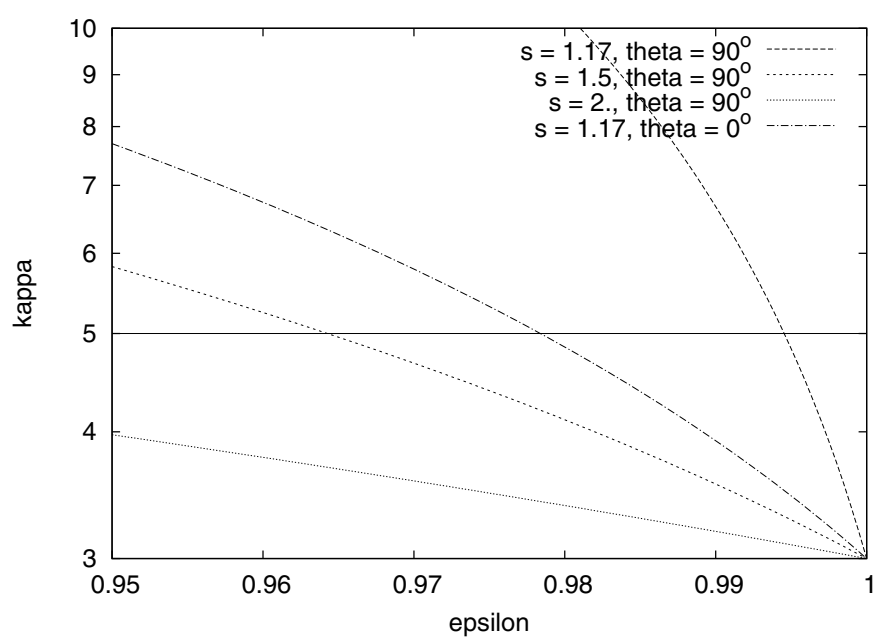

Fig. 5. Stable values for the power law index $\tilde{\kappa}$ as a function of the isotropisation parameter $\epsilon$ and shock parameters. The commonly observed index of $-\kappa=-5$ has been added to the figure.

fluctuations in the presence of a small (i.e. $\$ 5-10 \%$ ) temperature (i.e. pitch-angle) anisotropy. This result agrees well with the observational finding that the solar wind plasma in general may be considered as isotropic, although with just the small kind of fluctuations appearing in any realistic system because of local derivations from an ideal configuration.

\section{Conclusion}

We have demonstrated that a chain of bulk velocity jumps passing over a co-moving ion population under two specific conditions automatically leads to power law velocity distribution functions. The first condition is that the two particle invariants introduced in this paper are conserved at bulk velocity jumps, and the second that between the periods of the passage of two consecutive bulk velocity jumps the jump-induced initially anisotropic distribution is again isotropized rapidly enough by pitch-angle scattering. For these conditions, we have shown that a power-law distribution develops as an equilibrium ion distribution function with a spectral velocity power index of $-\kappa=-3$. Spectral indices of $-4.4 \geq-\kappa_{\text {obs }} \geq-6.6$ as often found by plasma analysers in heliospheric space plasmas (see e.g. Gloeckler 2003; Dayeh et al. 2009b; Desai et al. 2010) can be obtained in the framework of the aforementioned theory, if only partial, incomplete isotropisation of the ion distribution has taken place, before the next bulk velocity jump passage occurs. When incomplete isotropisation occurs within the period of one bulk regime passage, i.e. $\tau_{U}=L_{U} / \Delta U$, where the quantity $L_{U}$ is the one-dimensional extent of a mono-bulk regime, with only a percentage $\epsilon$ of ions becoming isotropized, the resulting power index will become larger. This covers the observationally found power indices at $0.95 \lesssim \epsilon \lesssim 1$, with $\tilde{\kappa} \simeq-5$ for an isotropisation degree of $\epsilon \simeq 0.99$.

On the other hand, we have to admit here that the above study was carried out for somewhat idealistic one-dimensional plasma flow conditions with no plasma flow divergence in the flow direction (i.e. no radially symmetric outflow). In reality, the solar wind plasma flow to first order is spherically symmetric with a flow divergence in the radial flow direction that leads to the magnetic or adiabatic cooling of ions (see Fahr 2007; Fahr $\&$ Siewert 2008) convected outwards to larger radial distances. The cooling of co-convected ions is due to both a differential 
bulk drift and the conservation of the magnetic moment in a flow with decreasing magnetic field magnitude and results in a velocity magnitude drift towards smaller particle velocities. As we have shown in Fahr \& Siewert (2008), this leads to (-5)-powerlaw ion distributions for ion velocities $v \leq U$ of pick-up ions injected at the injection border of $v=U$. If this effect were to be appropriately taken into account, then instead of a Maxwellian with $T_{\mathrm{f} 0}=m U^{2} / 3 \mathrm{~K}$ as taken in Eq. (41), one would start instead from an initial power-law function with $-\kappa=-5$, an initial upper velocity border of $v=U$ and a lower velocity border of $v \simeq U\left[1-(U / \Delta U)\left(L_{U} / r\right)\right]$, and then process this function by jump crossings.

In this context, we note that when studying the influence of bulk velocity fluctuations on ion distribution functions for the general Parker phase space transport equation when cooling processes are not taken into account, Zhang (2010) derived velocity power laws with a spectral index of $-\kappa_{Z}=-3$. In his study, it is suspected that adequate consideration of cooling processes in the framework of his theory would result in softer power-law spectra with $-\kappa \simeq-5$. This may allow the conclusion that bulk velocity fluctuations naturally lead to power laws, but only then to those with actually observed power indices, if the effects of ion cooling and incomplete isotropisation of distribution functions are properly taken into account, as we propose to study in the near future.

In the energizing process that has been studied in this paper, the energy arriving in the suprathermal power-law tails is generated by entropy production from the free energy sitting in the differential kinetic energies of succeeding bulk velocity jumps. The entropisation needed only occurs through isotropisation of the initially anisotropic distribution function (see Fahr \& Siewert 2010a) appearing immediately after jump passage where dynamical ion invariants are conserved. When no complete isotropisation is granted, off-equilibrium ion spectra with spectral velocity power indices of $\kappa_{v} \leq-3$ have to be expected.

The fully developed equilibrium that we have described in Sect. 4.4 is of course somewhat artificial, but represents the asymptotic state towards which the system would tend, if all competing processes (especially cooling processes) run slow. In our approximation, we have taken into account no cooling processes that, however, operate unavoidably in a radially diverging plasma flow and tend to steepen the spectra towards spectral indices $\kappa_{v} \leq-3$.

On the other hand, too high a pressure should not be a problem in the context of quasi-equilibrum ion power laws, since a natural upper velocity border always exists up to which the process we are studying here can operate. Only these ions can conserve their dynamical invariants at the crossing over the bulk velocity jump with subcritical velocities $v \leq v_{\mathrm{c}}$ (for a definition see Eq. (60)). At higher velocities $v \geq v_{\mathrm{c}}$, ions become free to escape by spatial diffusion from the jump structure and thereby do not conserve ion invariants. These particles thus stop participating in the pumping process due to bulk velocity jumps (i.e. compressive turbulence), thereby defining a roll-over and causing at supercritical ion velocities a fall-off of the ion spectrum to spectral slopes $\kappa_{v} \leq-5$ that keep the ion pressure finite.

Furthermore, at the end of this paper, we need to come back to the point raised in the beginning, namely that conservation of those particle invariants used in the aforementioned derivations can only be expected to hold for ions with subcritical velocities $v \leq v_{\mathrm{c}}=3 L_{U}^{2} \Delta U / \Lambda \Delta L$, i.e. ions that cannot escape from the jump structure by spatial diffusion while undergoing jumpinduced velocity transformation with conserved invariants. If passage times of the jump structure and diffusion times become comparable, i.e. if $\tau_{\mathrm{t}} / \tau_{\text {dif }}=v \Lambda \Delta L / 3 L_{U}^{2} \Delta U \simeq 1$, then ions can effectively escape from the jump structure without conserving the applied invariants. This then leads to an upper validity border of

$$
v \leq v_{\mathrm{c}}=3 U \frac{\Delta U}{U} \frac{L_{U}}{\Delta L} \frac{L_{U}}{\Lambda},
$$

where

$$
\begin{aligned}
& \frac{\Delta U}{U}=50 / 400=1 / 8, \\
& \frac{L_{U}}{\Delta L}=10 \mathrm{AU} / 10^{-2} \mathrm{AU}=10^{3}, \\
& \frac{L_{U}}{\Lambda}=10 \mathrm{AU} / 1 \mathrm{AU}=10,
\end{aligned}
$$

evaluates to $v_{\mathrm{c}} \simeq 3.75 \times 10^{3} \mathrm{U}$. This indicates that the theory presented here does not apply to high ion velocities with $v \geq v_{\mathrm{c}}$. At these energies, the theoretical approach presented by Fisk \& Gloeckler $(2006,2007,2008)$ instead applies which, however, as also in our equilibrum case, has to rely on completely isotropic ion distributions.

Acknowledgements. One of the authors, M. Siewert, is grateful to the Deutsche Forschungsgemeinschaft for financial support granted to him in the frame of the project Si-1550/2-1.

This research benefited greatly from discussions that were held at the meetings of the International Team devoted to understanding the -5 tails and ACRs that has been sponsored by the International Space Science Institute in Bern, Switzerland.

\section{Appendix A: Immediate re-shuffling of downstream ions back into the upstream regime}

Immediate re-shuffling of freshly generated slow-flow particles back into the fast bulk (i.e. triggered for instance by a very sharp $\boldsymbol{E}^{*}-\boldsymbol{E}$ - double-soliton structure), without prior mixing of velocity components, would lead to the velocity coordinate re-transformations

$$
\begin{aligned}
v_{\perp, \mathrm{f}}^{2^{\prime}} & =v_{\perp, \mathrm{s}}^{2} \sqrt{\cos ^{2} \alpha_{\mathrm{s}}+s_{\mathrm{sf}}^{2} \sin ^{2} \alpha_{\mathrm{s}}} \\
& =v_{\perp, \mathrm{f}}^{2} \sqrt{\cos ^{2} \alpha_{\mathrm{f}}+s_{\mathrm{fs}}^{2} \sin ^{2} \alpha_{\mathrm{f}}} \sqrt{\cos ^{2} \alpha_{\mathrm{s}}+s_{\mathrm{sf}}^{2} \sin ^{2} \alpha_{\mathrm{s}}}
\end{aligned}
$$

and

$$
\begin{aligned}
v_{\|, \mathrm{f}}^{2} & =v_{\|, \mathrm{s}}^{2} \frac{s_{\mathrm{sf}}^{2}}{\cos ^{2} \alpha_{\mathrm{s}}+s_{\mathrm{sf}}^{2} \sin ^{2} \alpha_{\mathrm{s}}} \\
& =v_{\|, \mathrm{f}}^{2} \frac{s_{\mathrm{fs}}^{2}}{\cos ^{2} \alpha_{\mathrm{f}}+s_{\mathrm{fs}}^{2} \sin ^{2} \alpha_{\mathrm{f}}} \frac{s_{\mathrm{sf}}^{2}}{\cos ^{2} \alpha_{\mathrm{s}}+s_{\mathrm{sf}}^{2} \sin ^{2} \alpha_{\mathrm{s}}} .
\end{aligned}
$$

Now, it follows from Eq. (5) that $B_{\mathrm{t} 1}^{\prime}=s_{\mathrm{fs}} s_{\mathrm{sf}} B_{\mathrm{t} 1}$; using Eq. (34) reduces this to $B_{\mathrm{t} 1}^{\prime}=B_{\mathrm{t} 1}$ and therefore, $B_{1}^{\prime}=B_{1}$. From this result, it immediately follows that

$$
\frac{B_{1}^{\prime}}{B_{2}}=\frac{B_{1}^{\prime}}{B_{2}} \frac{B_{2}}{B_{1}} \frac{B_{1}}{B_{2}}=\left(\frac{B_{2}}{B_{1}}\right)^{-1},
$$

or, using Eq. (8),

$$
\cos ^{2} \alpha_{\mathrm{s}}+s_{\mathrm{sf}}^{2} \sin ^{2} \alpha_{\mathrm{s}}=\frac{1}{\cos ^{2} \alpha_{\mathrm{f}}+s_{\mathrm{fs}}^{2} \sin ^{2} \alpha_{\mathrm{f}}} .
$$

This equation allows us to obtains the following complete identities from Eqs. (6) and (7)

$$
v_{\perp, \mathrm{f}}^{2^{\prime}}=v_{\perp, \mathrm{f}}^{2} \frac{\sqrt{\cos ^{2} \alpha_{\mathrm{f}}+s_{\mathrm{fs}}^{2} \sin ^{2} \alpha_{\mathrm{f}}}}{\sqrt{\cos ^{2} \alpha_{\mathrm{f}}+s_{\mathrm{fs}}^{2} \sin ^{2} \alpha_{\mathrm{f}}}}=v_{\perp, \mathrm{f}}^{2}
$$


H.-J. Fahr and M. Siewert: Isotropic ion distribution functions due to consecutive bulk velocity jumps

and

$$
\begin{aligned}
v_{\|, \mathrm{f}}^{2^{\prime}} & =v_{\|, \mathrm{f}}^{2} \frac{s_{\mathrm{sf}}^{2}}{\cos ^{2} \alpha_{\mathrm{f}}+s_{\mathrm{sf}}^{2} \sin ^{2} \alpha_{\mathrm{f}}} s_{\mathrm{fs}}^{2}\left(\cos ^{2} \alpha_{\mathrm{f}}+s_{\mathrm{fs}}^{2} \sin ^{2} \alpha_{\mathrm{f}}\right) \\
& =v_{\| 1}^{2},
\end{aligned}
$$

meaning that without stochastic or isotropizing processes on the downstream side the re-integrated ions exactly re-constitute the upstream distribution function, i.e. they are completely adiabatic and reversible.

If, however, prior to re-shuffling into the other bulk frame mixing between the two degrees of freedom parallel and perpendicular to $\boldsymbol{B}$, e.g. due to either wave generation or pitch-angle scattering, occurs, then the transformations undergo interesting irreversibilities, which we have studied in the main text.

\section{References}

Chalov, S. V. 2005, Adv. Space Res., 35, 2106

Chalov, S. V., Fahr, H.-J., \& Izmodenov, V. 1997, A\&A, 320, 659

Chew, G. F., Goldberger, M. L., \& Low, F. E. 1956, Proc. R. Soc. London A, 236, 112

Dayeh, M. A., Desai, M. I., Dwyer, J. R., et al. 2009a, ApJ, 693, 1588

Dayeh, M. A., Desai, M. I., Kozarev, K. A., et al. 2009b, AGU Fall Meeting Abstracts, A1492

Decker, R. B., Krimigis, S. M., Roelof, E. C., et al. 2005, Science, 309, 2020

Decker, R. B., Krimigus, S. M., Roelof, E. C., et al. 2008, Nature, 454, 67

Desai, M. I., Dayeh, M. A., \& Mason, G. M. 2010, Twelfth International Solar Wind Conference, 1216, 635

Diver, D. A. 2001, A Plasma Formulary for Physics, Technology and Astrophysics (Hoboken, New Jersey: John Wiley)

Erkaev, N. V., Vogl, D. F., \& Biernat, H. K. 2000, J. Plasma Physics, 64, 561

Fahr, H.-J. 2007, Ann. Geophys., 25, 2649

Fahr, H.-J., \& Siewert, M. 2007, ASTRA, 3, 21

Fahr, H.-J., \& Siewert, M. 2008, A\&A, 484, L1
Fahr, H.-J., \& Siewert, M. 2009, ApJ, 693, 281

Fahr, H.-J., \& Siewert, M. 2010a, ASTRA, 7, 1

Fahr, H.-J., \& Siewert, M. 2010b, A\&A, 512, A64

Fichtner, H., Le Roux, J. A., Mall, U., \& Rucinski, D. 1996, A\&A, 314, 650

Fisk, L. A. 1976, J. Geophys. Res., 81, 4633

Fisk, L. A., \& Gloeckler, G. 2006, ApJ, 640, L79

Fisk, L. A., \& Gloeckler, G. 2007, PNAS, 104, 5749

Fisk, L. A., \& Gloeckler, G. 2008, ApJ, 686, 1466

Forsyth, R. J., Balogh, A., \& Smith, E. J. 2002, J. Geophys. Res. (Space Physics), 107,1405

Gloeckler, G. 2003, in Solar Wind Ten, ed. M. Velli, R. Bruno, F. Malara, \& B. Bucci, AIP Conf. Ser., 679, 583

Gombosi, T. I. 1998, Physics of the Space Environment (New York: Cambridge University Press)

Hill, M. E., Schwadron, N. A., Hamilton, D. C., Di Fabio, R. D., \& Squier, R. K. 2009, ApJ, 699, L26

Infeld, E., \& Rowlands, G. 1990, Nonlinear Waves, Solitons and Chaos, ed. E. Infeld, \& G. Rowlands

Jokipii, J. R., \& Lee, M. A. 2010, ApJ, 713, 475

le Roux, J. A., \& Fichtner, H. 1999, J. Geophys. Res., 104, 4709

McComas, D. J., Barraclough, B. L., Funsten, H. O., et al. 2000, J. Geophys. Res., 105, 10419

McComas, D. J., Elliott, H. A., Schwadron, N. A., et al. 2003, Geophys. Res. Lett., 30, 100000

Parker, E. N. 1958, ApJ, 128, 664

Parker, E. N. 1965, Plan. Sp. Sc., 13, 9

Popecki, M., et al. 2010, Talk held at Tail-workshop at ISSI (Bern)

Richardson, J. D., Wang, C., \& Burlaga, L. F. 2003, Geophys. Res. Lett., 30, 2207

Richardson, J. D., Liu, Y., Wang, C., \& McComas, D. J. 2008, A\&A, 491, 1

Schwadron, N. A., Dayeh, M. A., Desai, M., et al. 2010, ApJ, 1386

Siewert, M., \& Fahr, H.-J. 2008, A\&A, 485, 327

Simunac, K. D. C., Kistler, L. M., Galvin, A. B., et al. 2009, Sol. Phys., 259, 323

Spatschek, K.-H. 1990, Theoretische Plasmaphysik (Stuttgart: Teubner Verlag)

Toptygin, I. N. 1983, Cosmic Rays in Interplanetary Magnetic Fields (Norwell/Mass.: D. Reidel Publ.)

Treumann, R. A., \& Baumjohann, W. 1997, Adv. Space Plasma Phys. (London: Imperial College Press)

Zhang, M. 2010, J. Geophys. Res., 115, A12102 